\title{
Implementing an Exact Estimation Approach of the Base Stock for the Periodic Review Policy Based on Fill Rate
}

\author{
Eugenia BABILONI, Ester GUIJARRO, Manuel CARDÓS \\ Universidad Politècnica de València, \\ Camino de Vera s/n, Valencia, Spain, \\ mabagri@doe.upv.es, esguitar@doe.upv.es, mcardos@doe.upv.es, soesmi@omp.upv.es
}

\begin{abstract}
Recent developments on the fill rate estimation in periodic review stock policies provide new techniques for setting up the stock policy parameters including exact procedures. However, the implementation of this method in real environments may be a difficult task due to issues such as the availability of demand data, the identification of an appropriate demand distribution pattern and the target fill rate established for each item according to a global target fill rate. This paper focuses on the modifications needed in the information management system of a company in order to implement an exact estimation procedure of the stock policies taking advantage of available data.
\end{abstract}

Keywords: Stock control, fill rate, data model, DSS.

\section{Introduction}

In inventory management two key questions should be answered: (1) when to place a replenishment order; and (2) how much should be order at each review period. When the inventory is managed by a base stock $(R, S)$ policy, answering the first question implies to determine the review period $R$, whereas the second question depends on the base stock level $S$. In practical environments, the review period is normally predetermined, so the real problem for managers consists in deter-mining the optimal S such that total inventory costs are minimized or some target customer service level is fulfilled.

Although the majority of the literature has focused on the criterion of minimization of costs, in real environments these costs are difficult to know and estimate, particularly costs incurred by not having stock to attempt the demand [1], [2]. For this reason, practitioners apply the service level criterion to establish the base stock level $S$, being the volume fill rate (denoted just fill rate or $\beta$ further on) the customer service measure most widely used. Furthermore, when managing inventories it is required to know how to proceed when an item is out of stock and a customer order arrives. This paper assumes that unsatisfied demand from the on hand stock is backordered.

The fill rate is defined as the fraction of demand that is immediately fulfilled from on hand stock. However, in order to avoid complex mathematical formulation, the most common approach to estimate it consists in computing the number of units short instead of computing directly the fulfilled demand per replenishment cycle. This approach (denoted by $\beta_{\text {Trad }}$ further on) calculates the complement of the quotient between the expected unfulfilled demand per replenishment cycle (also known as expected shortage) and the total expected demand per replenishment cycle, i.e.:

$\beta_{\text {Trad }}=1-\frac{E(\text { unfulfilled demand })}{E(\text { total demand })}$

In the literature, we find quite a number of works suggesting methods to estimate expression (1) in different contexts. However, few of those consider the periodic review systems, although these are arguably most realistic. One limitation of the available methods devoted to estimating $\beta_{\text {Trad }}$ in the $(R, S)$ system for the backordering case is that they usually assume continuous demand patterns, generally normally distributed. In this sense, [1], [3], [4] and [5] suggest methods to estimate it when demand is normally distributed whereas [6], [7] and [8] when demand follows any continuous distribution. The continuous assumption is the most common for modelling the demand since mathematical models are simplified. Unfortunately, in real environments the demand rarely is continuous, being the discrete demand the most common in practice [9].

Another approach to compute the fill rate consists in estimating directly the fraction of the fulfilled demand per replenishment cycle instead of determining the expected shortage, as follows: 


$$
\beta=E\left(\frac{\text { fulfilled demand }}{\text { total demand }}\right)
$$

[10] and [11] show that expression (1) and expression (2) are not equivalent. To the best of our knowledge, only [11] present exact estimation procedures for both expression (1) and (2) to compute the fill rate for periodic review systems under discrete demand context and backordering case. However the complexity of such methods makes difficult its implementation by practitioners. On the other hand, applying estimation procedures designed for continuous demand patterns to discrete demands leads to significant deviations that cannot be neglected as shown by [12]

Therefore, the exact estimation of the base stock for the periodic review policy based on the fill rate cannot be avoided but in practice it is feasible if and only if it is included as part of the inventory control module of the management information system. However the implementation of an exact estimation procedure is much more than just applying complex formulas but it also requires taking advantage of available data. The main purpose of this paper is to describe how the information management system of a company can be modified to cope with the main difficulties that arise when an exact approach is applied and how a Decision Support System (DSS) can help stock managers.

The rest of this paper is organized as follows. Section 2 points out some important technical problems related to the application of an exact estimation procedure in practice. However, in order to implement this kind of procedures the transactional system of the company should be modified (see Section 3) and a Decision Support System is also needed to help adopting the most appropriate decision on the stock management (see Section 4). Finally, Section 5 includes a summary of the main results provided in this paper. Appendix A includes the description and notation of a periodic review stock policy and Appendix B summarizes the derivation of $\beta_{\text {Trad }}$ and $\beta$ in a discrete demand context and its estimation procedures.

\section{Problems of Implementing Exact Estimation Procedures in Practice}

The estimation procedures described in Appendix $\mathrm{B}$ for $\beta_{\text {Trad }}$ and $\beta$ share some important characteristics that must be taken into account during their implementation. The first one is that these fill rate estimation procedures are used in practice to set the optimal base stock of the $(R, S)$ stock policy (see notation in Appendix A). Therefore an iterative procedure has to be conducted in order to find out the base stock level that guarantees the target fill rate using the lead time and the review period as inputs data. As a result, the base stock is set as the minimum value that reaches the target fill rate.

However, $\beta_{\text {Trad }}$ is an approximation of the real fill rate which tends to underestimate the real fill rate [11]. An important consequence of the underestimation behaviour is found when it is used to determine the base stock level. To illustrate the risk of using $\beta_{\text {Trad }}$ to set up the optimal $S$, we show an example of the evolution of $\beta_{\text {Trad }}$ and $\beta$ when increasing the base stock for a smooth demand modelled by a negative binomial with $r=4$ and $\theta=0.7$ (Figure 1 ). In this example if a target fill rate is set to $0.60, \beta_{\text {Trad }}$ leads to $S=5$ whereas in fact just $S=3$ is enough to reach the target fill rate. In this case, using $\beta_{\text {Trad }}$ to determine the base stock level leads to an unnecessary increase in the average stock level and thus the holding costs of the system. This type of deviation is especially relevant in industries in which the unit cost of the item is high and/or storage space is limited. Therefore, managers should be aware of the risk of using the fill rate approximation to set the base stock level.

However, the implementation of the exact fill rate $(\beta)$ in real environments presents a sort of problems related to the demand data, demand distribution and practical application among others [1], [13]. Regarding the demand data we found that in general the available data in companies are actually sales data, which are not equivalent. This fact can distort the knowledge of the real demand especially during periods of low service level because the demand of an item is not recorded when it is known that there is not stock.

Even if we assume that the demand data is available, a new problem arises when trying to use it to identify an appropriate demand distribution function and estimating its parameters. In the literature generally it is assumed that the distribution function is previously known as in Appendix B. Furthermore, the procedures for estimating $\beta_{\text {Trad }}$ 


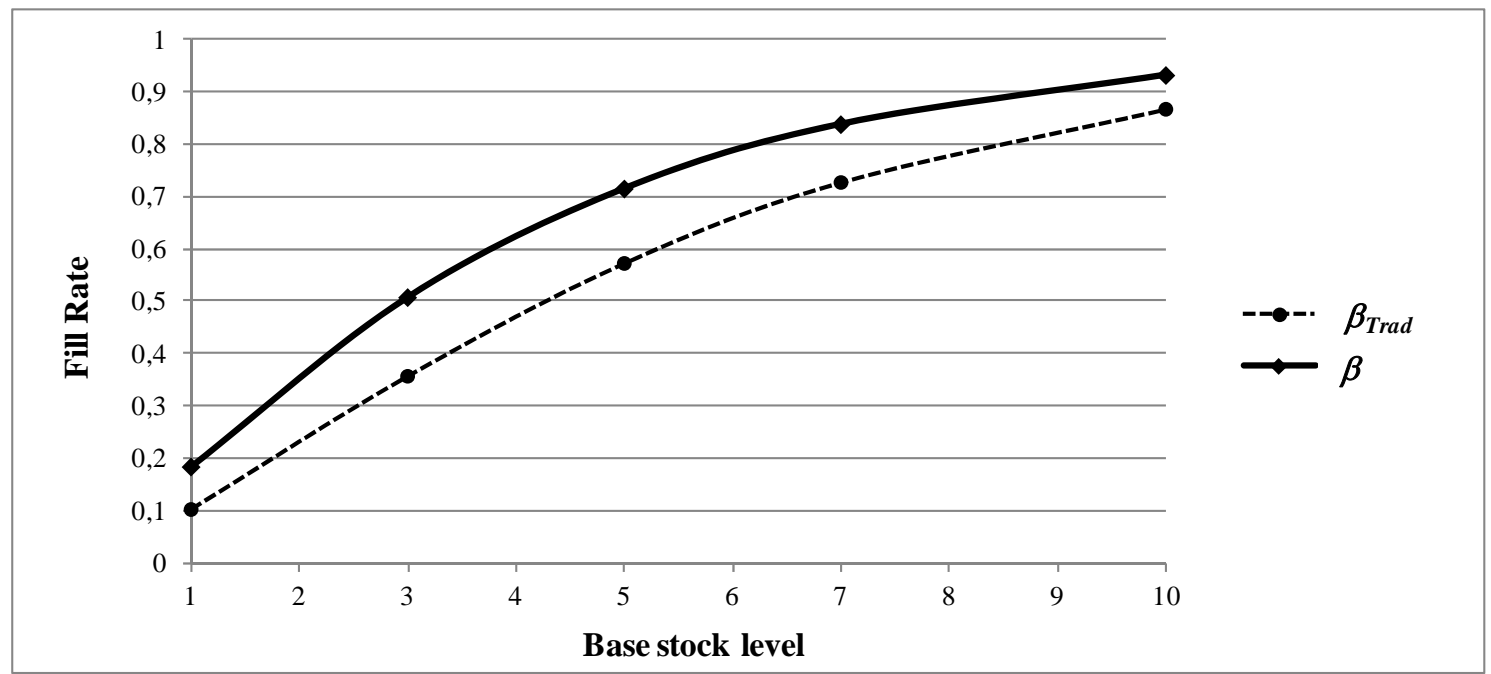

Figure 1. Comparison between $\beta_{\text {Trad }}$ and $\beta$ with negative binomial demand with $r=4$ and $\theta=0.7$ (smooth), $R=1$ and $L=1$

and $\beta$ can be applied to any known discrete demand pattern and therefore may cover the smooth, intermittent, erratic and lumpy categories proposed by [14]. From a practical point of view, in the discrete context the demand pattern is usually considered as Poisson or negative binomial distributed. This approach works fine except when the fraction of time periods without demand is very high, for example when just few days per year exhibit a non-zero demand. In this case, corresponding to lumpy and intermittent demand categories, the identification of the demand pattern and the estimation of its parameters becomes a difficult problem if some degree of statistical significance has to be achieved. For example, the demand of spare parts usually includes a large fraction of the items with this behaviour. As a consequence, two different scenarios have to be considered: (i) when the fraction of periods without demand is medium to low, and (ii) when the fraction of periods without demand is high.

\section{Implementing Exact Fill Rate Procedures in Practical Environments}

The first scenario proposed is determined by a medium to low fraction of periods without demand and can be managed with simple and well known techniques such as: (1) examining demand data; (2) determining the average and the standard deviation of demand; (3) proposing a demand pattern; and (4) estimating the base stock. Usually the demand pattern is selected as Poisson when the variance differs from the average in no more than a 10 per cent; if not so a negative binomial distribution is usually preferred.

On the other hand the second scenario is determined by demand data with high fraction of periods without demand. Obviously this is a more demanding situation as it may challenge the hypothesis of structural permanence because: (a) the number of non-zero demands during the usual data gathering period, typically a year, is very low, so that any statistical inference lacks statistical significance even for the demand pattern itself; and (b) in a try to gain statistical significance, data gathering period can be increased in order to collect more non-zero demand data, but this is only possible if the demand behaviour remains the same during the augmented period, but this is difficult to be assumed for items with scarce demand whose pattern is not well known. Therefore, in this second scenario, we propose an approach based on maintaining the usual data gathering period and just replacing the probability mass function by the observed empirical frequencies in the estimation procedures. Note that this approach avoids any assumption on the demand pattern and the need of statistical inference to determine the parameters of demand distribution although more demand data should be retained. Therefore this second scenario can be managed following these steps: (1) examining demand data; (2) proposing the observed empirical 
frequencies as the demand pattern; and (3) estimating the base stock.

The data model shown in Figure 2 supports the implementation of the estimation procedures for both scenarios and approaches. The data items are basically self-explanatory but some of them require some additional information: (a) demand accumulation period is the period of time used to aggregate the demand data, usually day or week, corresponds to period type, and establishes the time scale of the stock policy; (b) minimum demand periods indicates that at least this number of periods with nonzero demand should be accumulated, useful for items with a high ratio of no demand periods; and (c) maximum demand periods denotes the time span used to aggregate demand data. managing the stock of a set of items. In fact, target service levels are established for all items as a whole not for each one and the same occurs for the target inventory value. However stock policies are applied at an item basis, so both levels are necessarily linked by appropriate decisions.

Unfortunately some of these decisions require an expert to judge the specific characteristics involved, for example the item service level setting in order to fulfil the global service level. In practice, there is a relative consensus on using fixed service level for every item in each category of an ABC classification. But there is not on the number of categories should be used, the index to rank the items in order to develop the $\mathrm{ABC}$ classification (demand volume,

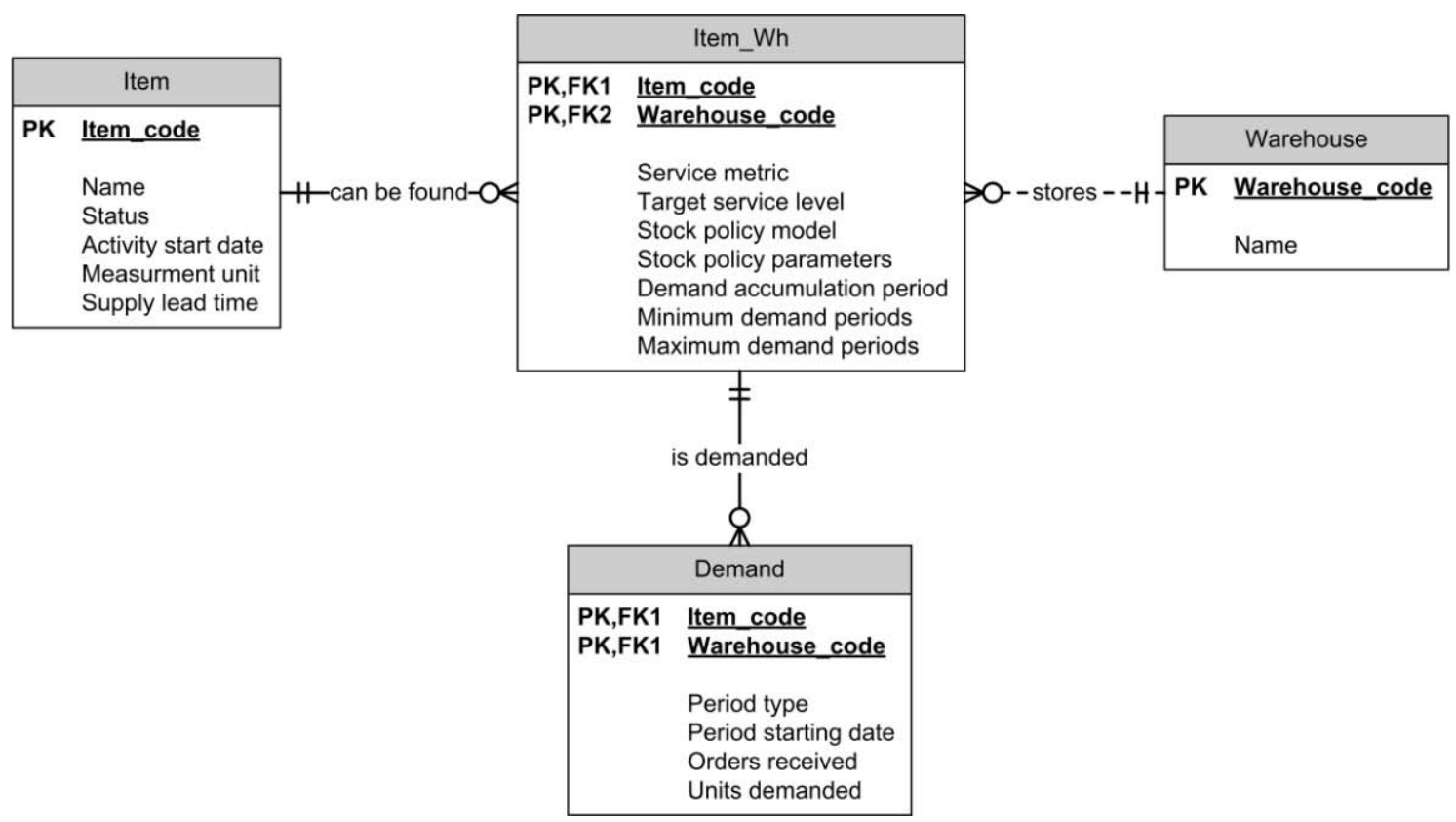

Figure 2. Data model for supporting the implementation of the procedures

It should be noted that different items may require different approaches and different data accumulation schemes, so the statistical data information usually provided by commercial information systems is not enough. Furthermore, usually the aggregation of these data is better performed by the transactional system due to the large amount of inventory transactions frequently involved.

\section{The Advisability of a Decision Support System}

Different points of view and a number of decisions have to be considered when demand value or cost related) or on the service level each category has to be set up [15], [16].

Therefore a Decision Support System (DSS) appears as a valuable tool for stock managers to explore repeatedly over the time the consequences of stock decisions according to the final purpose of the company, for example the achievement of a global fill rate while minimizing the total inventory value. Figure 3 shows the scheme of the proposed DSS and its main constituent elements. Note that targets on service and inventory levels are a very important part of the input data, but also the historical data basically obtained using the data model explained above in Section 3. 


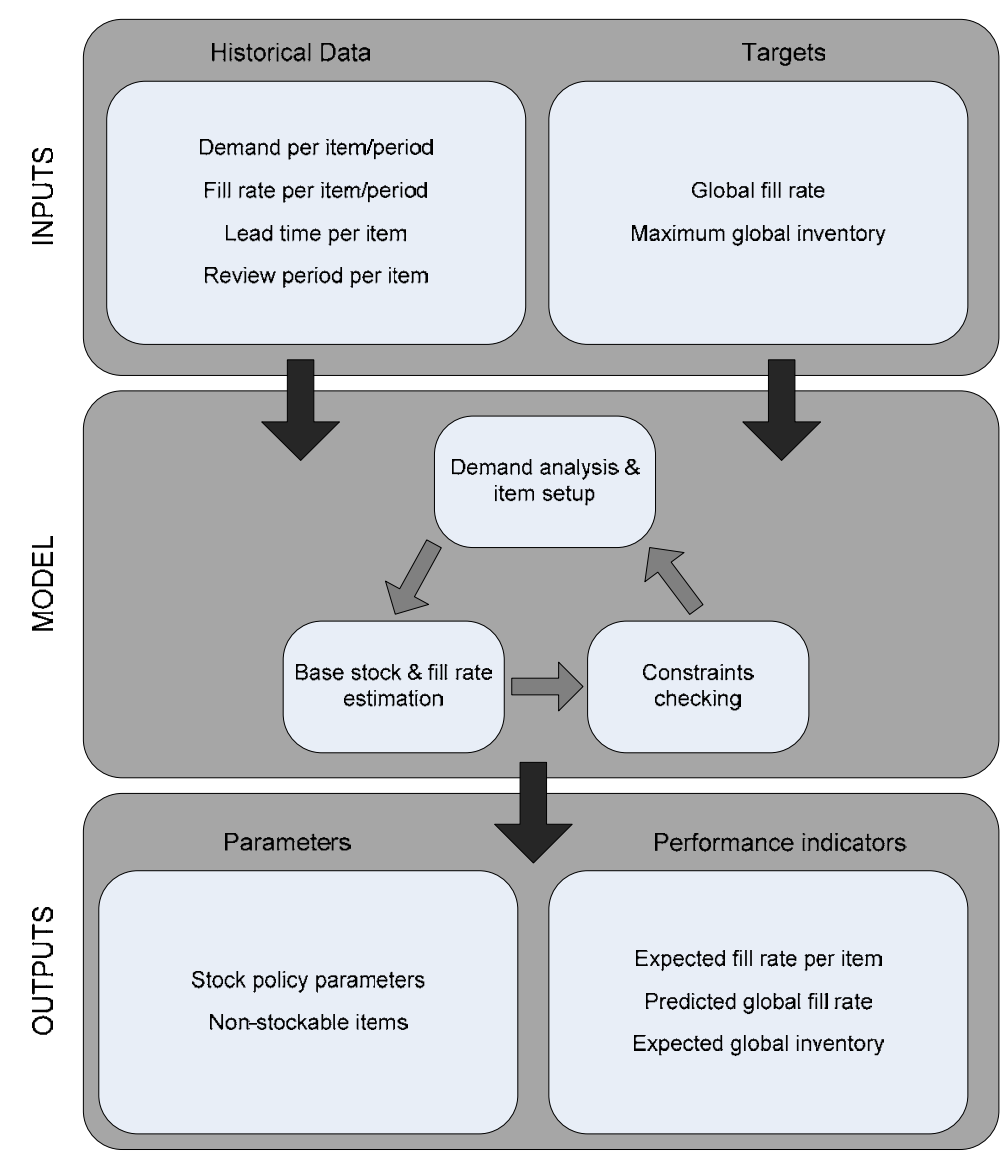

Figure 3. Scheme of decision support system

Once input data are available, in the first module (inputs) a detailed analysis of demand data should be performed in order to identify its distribution function and to estimate its parameters, as explained in Section 3. Moreover, predicted fill rates and inventory levels have to be compared against the actual ones on an item and category basis, so that trend changes could be detected. Additionally, a specific service level has to be assigned to every item based on the expertise of the stock manager. The second module of the model is base stock estimation based on the estimation of the fill rate exposed in Appendix B and whose exact estimations for any discrete distribution provides a sound foundation to assess the impact of different decisions on stock policies. Finally (outputs module), resulting calculations have to be checked to assure the effective fulfilment of the service and inventory constraints so that service levels may be adjusted and more importantly some items may be set as non-stockable based on managerial or technical decisions (in some cases a base stock of just one unit may deal to a fill rate of almost $100 \%$ because of its scarce demand). Obviously this is an iterative what-if process searching for an adequate trade-off between service and inventory levels.

Two sets of outputs should be provided: performance indicators and item parameters. Based on the first set, stock decisions may be approved and monitored so that predicted performance may be compared to actual performance the next time this process is performed. On the other hand, item parameters should be used by the transactional system in order to apply the stock decisions that have been approved.

\section{Summary}

This paper faces the consequences in practical environments of implementing an exact estimation procedure for the $(R, S)$ inventory policy based on determining the optimal base stock $S$ given a target fill rate. Fill rate measures the fraction of demand that is satisfied directly from shelf. In the literature, this service metric is usually computed by using the traditional approach $\left(\beta_{\text {Trad }}\right)$, which computes the fill rate by estimating the ratio between the expected unfulfilled demand and 
the total expected demand per replenishment cycle, instead of computing directly the expected fraction of fulfilled demand. However, computing the fill rate as $\beta_{\text {Trad }}$ tends to underestimate the real fill rate which has important consequences when it is used to determine the base stock level of the inventory policy (Figure 1). Furthermore, the methods found in the literature to estimate $\beta_{\text {Trad }}$ apply only under continuous demand distribution, generally normally distributed. In real environments, however, demand is usually discrete and therefore these expressions are not useful. Recently [11] propose a new exact expression $(\beta)$ to compute the fill rate directly as the fraction of fulfilled demand under discrete demand context.

But the implementation of an exact method of this kind in real environments may be a difficult task for practitioners. As Section 2 details, the application of exact procedures in an information system presents some problems such as the availability of demand data or the estimation of an appropriate demand distribution. In this sense, Section 3 describes two different scenarios: (i) when the fraction of periods without demand is medium to low, and (ii) when the fraction of periods without demand is high. This paper proposes a data model (Figure 2) that can be used to improve data gathering to cope with both scenarios. Additionally a Decision Support System (DSS) would be worthy to help stock managers assess different approaches in the search of the required service level while minimizing the inventory value.

\section{Acknowledgements}

This research was part of a project supported by the Universitat Politècnica de València, with reference number PAID-06-11/2022.

\section{REFERENCES}

1. HADLEY, G., T. WhITIN, Analysis of Inventory Systems, Prentice-Hall, Englewood Cliffs, NJ, 1963.

2. SILVER, E. A., Operations-Research in Inventory Management - A Review and Critique, Operations Research, vol. 29, no. 4, 1981, pp. 628-645.

3. JOHNSON, M. E., H. L. LEE, T. DAVIS, R. HALL, Expressions for Item Fill Rates in Periodic Inventory Systems, Naval Research Logistics, vol. 42, no. 1, 1995, pp. 57-80.

4. SILVER, E. A., D. F. PYKE, R. PETERSON, Inventory Management and Production Planning and Scheduling, Wiley, New York, 1998.

5. SILVER, E. A., D. P. BISCHAK, The Exact Fill Rate in a Periodic Review Base Stock System under Normally Distributed Demand, Omega International Journal of Management Science, vol. 39, no. 3, 2011, pp. 346-349.

6. SOBEL, M. J., Fill Rates of Single-stage and Multistage Supply Systems, Manufacturing and Service Operations Management, vol. 6, no. 1, 2004, pp. 41-52.

7. ZHANG, J., J. ZHANG, Fill Rate of Single-stage General Periodic Review Inventory Systems, Operations Research Letters, vol. 35, no. 4, 2007, pp. 503-509.

8. TEUNTER, R. H., Note on the Fill Rate of Single-stage General Periodic Review Inventory Systems, Operations Research Letters, vol. 37, no. 1, 2009, pp. 67-68.

9. SWAMINATHAN, J. M., J. G. SHANTHIKUMAR, Supplier Diversification: Effect of Discrete Demand, Operations Research Letters, vol. 24, no. 5, 1999, pp. 213-221.

10. GUIJARRO, E., M. CARDÓS, E. BABILONI, On the Exact Calculation of the Fill Rate in a Periodic Review Inventory Policy under Discrete Demand Patterns, European Journal of Operational Research, vol. 218, no. 2, 2012, pp. 442-447.

11. BABILONI, E., E. GUIJARRO, M. CARDÓS, S. ESTELLÉS, Exact Fill Rates for the (R, S) Inventory Control with Discrete Distributed Demands for the Backordering Case, Informatica Economica, vol. 16, no. 3, 2012, pp. 19-26.

12. GUIJARRO, E., E. BABILONI, M. CARDÓS, J. M. ALBARRACÍN, On Fill Rate Approximations in Periodic Review Systems for Discrete Demand, Industrial Engineering: Innovative Networks, Springer-Verlag London Limited, 2012.

13. WADHWA, S., B. A. PRAKASH, Service Performance of Some Supply Chain 
Inventory Policies Under Demand Impulses, Studies in informatics and Control, vol. 17, no. 1, 2008, pp. 45-56.

14. SYNTETOS, A. A., J. E. BOYLAN, J. D. CROSTON, On the Categorization of Demand Patterns, Journal of the Operational Research Society, vol. 56, no. 5, 2005, pp. 495-503.

15. VISWANATHAN, S., R. BHATNAGAR, The Application of ABC Analysis in Production and Logistics: An Explanation for the Apparent Contradiction, International Journal of Services and Operations Managements, vol. 1, no. 3, 2005, pp. 257-267.

16. TEUNTER, R. H., M. ZIED BABAI, A. A. SYNTETOS, ABC Classification: Service Levels and Inventory Costs, Production and Operations Management, vol. 19, no. 3, 2010, pp. 343-352.

\section{Appendix A. Description of the Inventory Policy and Notation}

In this paper, the inventory is controlled using the traditional periodic review, base stock $(R$, $S)$ policy which places a replenishment orders every $R$ units of time to raise the inventory position up to the base stock $S$. The replenishment order is received $L$ periods after being launched.

Figure 1 shows an example of the evolution of the on hand stock (stock that is physically on shelf), the net stock (on hand stock backorders) and the inventory position (on hand stock + stock on order - backorders) for the backordering case.

The notation used in Figure 1 and in this paper is as follows:

$S=$ base stock (units),

$R=$ review period corresponding to the time between two consecutive reviews and replenishment cycle corresponding to the time between two consecutive deliveries (time units), $L=$ lead time for the replenishment order (time units),

$\mathrm{OH}_{t}=$ on hand stock at time $t$ (units), $I P_{t}=$ inventory position at time $t$ (units),
$N S_{t}=$ Net stock at time $t$ (units),

$D_{t}=$ total demand during $t$ consecutive periods (units),

$f_{t}(\cdot)=$ probability mass function of $D_{t}$,

$F_{t}(\cdot)=$ cumulative distribution function of $D_{t}$,

We assume that: (i) demand process is discrete, stationary and i.i.d. in successive periods; (ii) demand during a period is fulfilled with the on hand stock at the beginning of the period; (iii) unfulfilled demand in a period is backordered and filled as soon as possible; (iv) the review time $R$ is constant and given; and (v) the lead time $L$ is fixed and known.

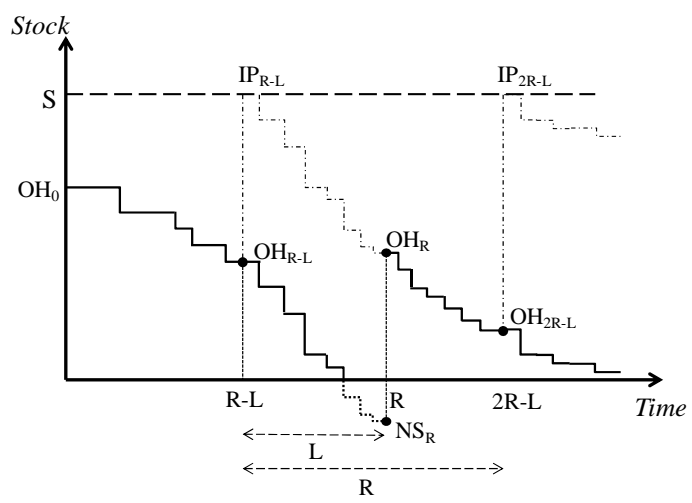

Figure 1. Example of the evolution of a $(R, S)$ system (backordering case)

\section{Appendix B. Estimation of the Fill Rate in a Discrete Demand Context}

\section{B1. Derivation of an exact method to compute $\boldsymbol{\beta}_{\text {Trad }}$}

Traditionally the fill rate has been computed as the complement of the ratio between the expected unfulfilled demand and the expected demand per replenishment cycle as shown in expression (1). As [11] explain the expected demand can be straightforwardly computed so all that is left to compute is the expected unfulfilled demand per replenishment cycle. Obviously, the unfulfilled demand depends on both the amount of demand during the cycle, $D_{R}$, and the available stock at the beginning of the cycle. Then, if at the beginning of the cycle there is not stock on shelf to satisfy any demand $\beta_{\text {Trad }}$ is equal to zero.

On the other hand if the net stock at the beginning of the cycle is positive $\left(N S_{0}>0\right)$, the shortage is equal to the difference between the $N S_{0}$ and the amount of demand that exceed $N S_{0}$ 
during that cycle. By definition, the net stock when positive can be from 1 to $S$, and hence:

$$
\begin{aligned}
& E(\text { unfulfilled demand })= \\
& =\sum_{N S_{0}=1}^{S} P\left(N S_{0}\right) \cdot \sum_{D_{R}=N S_{0}+1}^{\infty}\left(D_{R}-N S_{0}\right) P\left(D_{R}\right)
\end{aligned}
$$

Since the probability of every net stock level at the beginning of the cycle is $\mathrm{P}\left(N S_{0}\right)=\mathrm{P}\left(D_{L}=S\right.$ $\left.N S_{0}\right)=f_{L}\left(S-N S_{0}\right)$, the $\beta_{\text {Trad }}$ can be estimated as:

$$
\beta_{\text {Trad }}=1-\frac{\sum_{N S_{0}=1}^{S} f_{L}\left(S-N S_{0}\right) \cdot \sum_{D_{R}=N S_{0}+1}^{\infty}\left(D_{R}-N S_{0}\right) f_{R}\left(D_{R}\right)}{\sum_{D_{R}=1}^{\infty} D_{R} \cdot f_{R}\left(D_{R}\right)}
$$

Note that this expression is able to cope to any discrete distribution function.

\section{B2. Derivation of an exact method to compute $\beta$}

The fill rate is defined as the fraction of demand that is immediately fulfilled from shelf and therefore cycles that do not show any demand should not be taken into account. According to [10] to derive an exact method to compute $\beta$ over different demand patterns it is necessary to include explicitly the condition of having positive demand during the cycle. Following this reasoning, [11] propose an exact expression to compute the fill rate as expression (2) when demand follows any discrete demand distribution. In this case, authors focus on calculating the satisfied demand instead of the number of units short.
Then, positive demand during a cycle can be: (i) lower or equal than the net stock at the beginning of this cycle, i.e. $D_{R} \leq N S_{0}$, and therefore the fill rate will be equal to 1 ; or (ii) greater than the net stock, i.e. $D_{R}>N S_{0}$, and therefore the fill rate will be the fraction of that demand which is satisfied by the on hand stock at the beginning of this cycle. Hence

$$
\begin{gathered}
\beta\left(N S_{0}\right)=P\left(D_{R} \leq N S_{0} \mid D_{R}>0\right)+ \\
\sum_{D_{R}=N S_{0}+1}^{\infty} \frac{N S_{0}}{D_{R}} \cdot P\left(D_{R} \mid D_{R}>0\right)
\end{gathered}
$$

where the first term indicates (i) and the second term indicates (ii). Rewriting expression (5) through the probability mass and cumulative distribution functions of demand, $f_{t}(\cdot)$ and $F_{t}(\cdot)$, respectively, results in

$$
\begin{gathered}
\beta\left(N S_{0}\right)=\frac{F_{R}\left(N S_{0}\right)-F_{R}(0)}{1-F_{R}(0)}+ \\
\sum_{D_{R}=N S_{0}+1}^{\infty} \frac{N S_{0}}{D_{R}} \cdot \frac{f_{R}\left(D_{R}\right)}{1-F_{R}(0)}
\end{gathered}
$$

and applying expression (6) to every positive net stock level at the beginning of the period, the exact method to estimate $\beta$ when demand follows any discrete distribution function results as follows:

$$
\begin{aligned}
& \beta=\sum_{N S_{0}=1}^{S} f_{L}\left(S-N S_{0}\right) . \\
& \sum_{N S_{0}=1}^{S}\left\{\frac{F_{R}\left(N S_{0}\right)-F_{R}(0)}{1-F_{R}(0)}+\sum_{D_{R}=N S_{0}+1}^{\infty} \frac{N S_{0}}{D_{R}} \cdot \frac{f_{R}\left(D_{R}\right)}{1-F_{R}(0)}\right\}
\end{aligned}
$$

